\title{
Viabilidad del proyecto de la nueva derecha en El Salvador (segunda parte)
}

Carlos F. Hernández M.

En el capitulo cuarto se presentó el modelo que propone la nueva derecha a nivel económico y político-social, en los que se sostiene, como premisa central, que la crisis que se vive no es del sistema sino de la dirección del mismo; por lo tanto, la solución debe apuntar en el sentido de un nuevo proyecto de conducción del estado salvadoreno, que permita la superación de la crisis a nivel económico, político y social. En tal marco conceptual se trata de ubicar el origen de la crisis, desestimando en forma a priori las causas estructurales de la misma, lo que puede conducir a que se cometa el error de llegar a deducciones, sustentadas solo en el marco de razonamiento de la lógica formal y expresar conclusiones respecto de la solución de la crisis propuesta en los modelos que podrían considerarse no valederas, si nos atenemos al hecho de que se propone una solución, no sustentada en las verdaderas causas que la originan, pero, juzgar asi la solución propuesta, serla incurrir en similar nivel de error de apreciación del actor que las ofrece. Por lo tanto, la viabilidad de los modelos que propone la nueva derecha, se tratará de abstraer el análisis que se efectuará en un marco global de contrastar:

1. El poder que posee el actor nacional que propone tales modelos.

2. El objetivo central que persigue: defensa y reproducción del sistema capitalista.

3. El actor y actores del sistema internacional, que apoyan la implementación en el pais de tales modelos. 
4. El concenso que es capaz de generar tales modelos en las diferentes fuerzas políticas sociales salvadorenas.

Pasemos a tratar de contrastar cada uno de estos atributos, con las caracteristicas de la realidad que rodean al actor que propone los modelos aludidos, que, para nuestro caso particular, dicho actor se materializa en FUSADES. En tal orden, cabe preguntar ¿qué nivel de poder posee en la actualidad FUSADES? Para contestar a esta interrogante, es prudente aclararnos dos cosas: la primera, ¿cuáles son las manifestaciones empiricas del poder? y la segunda, ¿dónde se ubican las causas estructurales del origen del poder? En relación a las manifestaciones empiricas del poder, éstas se pueden resumir en: capacidad de influir en la acción y decisiones de la sociedad, capacidad real del uso de la coacción y capacidad real de conservar y/o modificar las instancias del estado.'

FUSADES, por ser una institución que agrupa a los intelectuales orgánicos, industriales, comerciantes y financistas de la nueva derecha $^{2}$, se puede considerar que el nivel de poder que maneja principalmente, se concreta en la capacidad de influir en las acciones y decisiones, de las instituciones de la sociedad civil y política, que por otras razones, además de las ya senaladas, le han permitido tener control de dichas instituciones. Es este marco de control institucional, que, en alguna medida, facilitará a los integrantes de la nueva derecha, influir en el sentido que deberán apuntar la organización y acción de las instituciones de la sociedad civil y política, a fin de orientar a la sociedad salvadorena en forma global, en la consecución de sus intereses, tanto a nivel económico, como político-social e ideológico.

En este orden que implica para los miembros de esta fuerza politico-social, ser accionistas, ocupar cargos directos o puestos gerenciales de tales instituciones de la sociedad civil y algunos altos cargos en la administración pública, sin que esto último se interprete como una de las metas que persigue la nueva derecha, pues se debe tener presente, como ya ha sido expuesto, que este actor no persigue el control directo del poder político, sino penetrarlo ideológicamente y que se convierta en un ejecutor de sus modelos, independientemente de la fuerza política que lo ejerza. Este marco que describe obmo la nueva derecha, a través de sus miembros, ejerce el control de diversas instituciones, es to que le permite influir en las políticas de acción de tales organismo y de esa forma ejercer el poder, que bien se puede considerar como una expresión de hegemonia por dirección en tales instituciones.

En lo que respecta a las otras expresiones empíricas de poder, se 
puede sostener, que en forma indirecta y por medio de diferentes mecanismos que pueden cubrir, desde simple publicación de manifiestos, publicaciones periódicas, elaboración de "estudios", formación de profesionales y las instituciones mismas de la nueva derecha, influyen en la orientacion del ejercicio real del poder, por medio de justificar y/o solicitar el uso de la coacción en contra de unos o en favor de determinados actores sociales ${ }^{3}$ a fin de defender al capitalismo y garantizar su reproducción, en forma genérica. De esta misma forma y utilizando los mismos mecanismos, es factible que pueda influir en la conservación y en la modificación de las estructuras del estado, siempre que esto viabilice la ejecución de los modelos que propone, en el marco del capital genérico. En resumen, se puede decir que la nueva derecha, la variable de poder que controla y ejerce, se ubica en la capacidad de influir en las decisiones y acciones a nivel social, en virtud del control que, en alguna medida, ejerce sobre las instituciones de la sociedad civil o instituciones comúnmente llamadas privadas.

En cuanto se refiere al origen estructural del poder que maneja la nueva derecha salvadorena, materializada en los integrantes de FUSADES, este se levanta de la riqueza que posee e ingreso que percibe, de las empresas productivas que controlan, ${ }^{4}$ situación que los pone en una posición ventajosa de tomar las decisiones de esas instituciones privadas y de esta manera, orientar e influir el proceso de toma de decisiones a nivel social, proceso que, al cumplirse le permitirá ejercer un nivel de hegemonia por dirección, cosa que está de acuerdo con el planteamiento que viene manejando de no controlar en forma directa el poder político, sino penetrarlo ideológicamente, a fin de que tome como propios los modelos propuestos y los ejecute. En el entendido de que es la forma racional y mejor de superar la crisis. De forma tal que se puede decir, a manera de resumen, que los modelos propuestos por el actor que tratamos, no están en discución, sino que éstas se centran en la fuerza político-social que los debe ejecutar en la práctica. En este sentido, da la impresión de que esta fuerza asume una aparente posición de neutralidad política y que sus modelos propuestos obedecen a una preocupación eminentemente técnica y al vivo deseo de superar la crisis. ${ }^{5}$

Fines aparentemente desinteresados, que encubren con mucha sutileza, el verdadero objetivo que persigue la nueva derecha, el cual radica esencialmente en que los modelos que ha propuesto, servirán para establecer una defensa organizada del sistema y garantizar asl su supervivencia y reproducción, en el marco de un capitalismo genérico, que no descarta el sacrificio de los elementos más conservadores de la derecha tradicional, elementos que con su actitud reaccionaria, ame- 
nazan la estabilidad y reproducción del sistema, conducta que, en alguna medida, ha contribuido en la generación de la actual crisis.

Consiguientemente los modelos pretenden alcanzar una modemización del sistema, referida al esquema tradicional ya fracasado, que en lo económico se expresó por medio de una producción primaria monocultivista para la exportación y a nivel industrial en un esquema de industrialización de sustitución de importaciones, a fin de abastecer con tales manufacturas, la demanda del mercado común centroamericano. En este orden de cosas, la nueva derecha juzga que el fracaso de tal modelo productivo agricola $e$ industrial, se encuentra en lo restringido de la producción y de los mercados. Eso se vuelve evidente, desde el momento que los planificadores de los modelos aludidos, llegan a considerar que la viabilidad de los mismos, en su mayor parte, se localiza en la capacidad de poder diversificar las exportaciones agricolas y los mercados externos, ${ }^{8}$ asi como también a nivel interno, en la generación de empleo, ya que tales exportaciones deberán ser intensivas en trabajo o al menos incrementar el nivel de ocupación agricola actual.

De lo anterior se puede juzgar, que la nueva derecha está proponiendo un modelo económico de crecimiento hacia afuera, rechazando implicitamente todo esquema de crecimiento hacia dentro, debido a que neceriamente tendría que pasar por la creación de un mercado intemo de consumidores, asl como también ejecutar en la práctica cambios en la actual distribución de la riqueza y del ingreso. Por lo tanto, se puede llegar a sostener que tal modelo implicará costos como los siguientes: primero, un crecimiento de la dependencia, referida al pals que se convertirá en el mejor mercado. FUSADES considera que tendrá que ser E.U.A. Segundo, deberá tenerse claro que dicho modelo implica, el mantenimiento de la actual situación estructural, que no es favorable a los sectores sociales de ingresos medios y bajos. Por lo tanto, es de suponer que se ampliará la brecha que separa la clase fundamental de la subalterna. Tercero. Este mayor deterioro de las condiciones de vida del sector laboral, se manifestará por medio de un incremento del conflicto social, fenómeno que empíricamente se ha expresado de un mayor descontento del sector laboral, a través de un agravamiento de las relaciones obreropatronales de junio al —noviembre de 1989_, protestando por las medidas de política económica del gobierno - liberalización de los precios-, que sólo tiende a favorecer al sector de la empresa privada.? Cuarto. Es de suponer que en este esquema de agravamiento del conflicto social, ocasionado en parte por la ejecución de una de las medidas económicas propuestas por la nueva derecha - liberalización 
de los precios- contribuirá a profundizar la polarización de las fuerzas políticas y del conflicto político-social, cosa que en alguna medida le resta viabilidad al modelo económico de FUSADES.

En el orden político, el modelo de gobiemo oligárquico-militar, que dio origen a un sistema político excluyente, explicado en capítulo anterior, también deberá ser sujeto de un proceso de modernización, fenómeno que tendrá que expresarse, a través de lo que la nueva derecha llama "Democracia plebiscitaria", en la cual deberá de existir todos los elementos formales de la democracia, elementos que, dado el nivel de radicalización imperante en la actualidad y la tendencia de dominación futura, de tal posición en las fuerzas político-sociales, le restará viabilidad al modelo político-social que propone la nueva derecha, sobre todo en lo que se refiere a la creación y fortalecimiento de las instituciones democráticas, que se deberán expresar por medio del respeto a los derechos humanos, políticos, económicos y sociales, de todos los salvadorenos, estadio social que debería facilitar el logro de la estabilidad política y la paz, en el corto plazo, apuntando a crear las condiciones que permitan llegar al desarrollo de la democracia en el mediano y largo plazo. El problema actualmente radica en que, cuando se polarizan las fuerzas políticas, endurecen sus posiciones, esto es precisamente to que está sucediendo en nuestra sociedad. En tal sentido, los proyectos propuestos, serán juzgados en forma negativa, mayormente por venir de la fuerza opuesta, que, por su contenido, situación que al actor que analizamos, conducirá a restarle aceptación a los modelos que propone en lo económico y político-social. Esto no debe juzgarse en el marco simplista de que lo propuesto en sus modelos es malo o bueno. Tales modelos deberán ser vistos bajo la óptica del beneficio social que genere su ejecución, en un segundo nivel, si en realidad su puesta en marcha, permitirá la superación de las condiciones que impiden alcanzar el desarrollo en los órdenes económicos, político y social, tal como lo sostiene la nueva derecha o en la práctica este es un argumento expuesto, con la finalidad de encubrir el verdadero propósito: que se ubica en mantener la actual injusticia y violencia estructural en el pais.

En relación al objetivo central que persigue la nueva derecha, éste consiste esencialmente en una estrategia global de: defensa y reproducción del sisterna capitalista, con la finalidad de poder seguir ejerciendo la hegemonia en función de los intereses de la clase fundamental, aunque esto se debe ver apuntando a favorecer, a la fracción progresista de la misma, que se identifica ideológicamente o es parte integrante de la nueva derecha. En tal orden de objetivos se vuelve evidente que los enunciados, que aparecen en los modelos, como son 
los referentes a un desarrollo de la democracia y sus instituciones; en el nivel polltico, la nueva derecha los utiliza con mucha sutileza, para encubrir la hegemonla que pretende ejercer al sustituir en tal función a la derecha tradicional. En lo referente al crecimiento, tendiente a llegar a un desarrollo económico posterior, mimetiza con habilidad las desigualdades que se originan en la conservación de las relaciones sociales de producción capitalista, en la forma histórica que se han expresado en nuestra sociedad, aunque dicho actor propone una modernización de las mismas, cosa que debe ser observada en el marco de cumplir con los principios de incremento de la rentabilidad, productividad y eficiencia, que apuntan hacia una mayor apropiación del excedente económico generado en la producción, ya sea en forma absoluta y/o relativa.

A partir de este marco, es que se puede analizar la viabilidad del modelo, en función del objetivo particular expuesto, en to que se refiere al mantenimiento del sistema. A este respecto, pareciera ser que buena parte de las fuerzas político-sociales de la nación se aproximan a un consenso, en el sentido de que todas aceptan to que se ha dado en denominar como "Economia Social de Mercado", subyaciendo en el fondo de esto, el convencimiento de la adecuada combinación del principio económico de la eficiencia mediante la competencia, con el objetivo político del equilibrio social, ${ }^{\theta}$ que debe existir en la sociedad. Sin embargo, parece ser también que este concepto de economia social de mercado, es tomado con diferencias de connotación por los agentes económicos, cada uno viéndolo en el sentido de mayor beneficio a sus particulares intereses, y no en la perspectiva de presentar una alternativa de solución a la crisis económica-política del estado salvadoreno. Esto vuelve complejo el panorama. Sin embargo, se puede llegar a sostener que los sectores sociales de ingresos medios, dado el patrón histórico de conducta que siempre han seguido, apoyarán el modelo económico y político propuesto por FUSADES. La incertidumbre surge en cuanto a cómo será la respuesta al modelo que expresarán los sectores sociales de ingresos bajos - sector laboral, obrero y campesino- los que, en alguna medida, ya han manifestado su oposición a algunas de las medidas económicas implementadas al presente, que forman parte del modelo económico propuesto por FUSADES, como to es específicamente la liberación de los precios. En lo que se refiere a la respuesta de la derecha tradicional, es de esperar que la efectuará con reticencia y atraso. Pero, en última instancia, apoyando al modelo de la nueva derecha, ya que, en el marco de los mismos, sus intereses económicos y político-sociales, continuarán ocupando una posición de privilegio y además, convencida de que el 
modelo agroexportador monocultivista, tendrá vida por un buen periodo, situación que en alguna medida podrá permitir formar las estrategias que faciliten la recuperación de la hegemonla de la que haya sido desplazada. Esto es lo propio de la lucha por el poder y seria ingenuo pensar que la derecha tradicional, al ser desplazada de su posición de hegemonia, no intente recuperarla, aceptando tal situación conformistamente.

Finalmente, se puede decir que la viabilidad del objetivo analizado, en el marco del modelo global propuesto por la nueva derecha salvadorena, dada las actuales circunstancias de polarización de las fuerzas político-sociales, más que ser aceptado por consenso, será impuesto por coacción.

En to que se refiere a los actores del sistema internacional, que apoyan la implementación de los modelos que propone FUSADES, tenemos a la nueva derecha estadounidense, cuyas expresiones se plasman concretamente en los documentos de las comisiones Kissinger, Santa Fe, Sanford y Plan de la Cuenca del Caribe, documentos por medio de los cuales, se trata de orientar la forma de expresión de la política exterior estadounidense hacia los paises de la región, en apoyo directo de la nueva derecha de los diferentes estados, cobrando expresión concreta a través de: asesoria, becas para la formación de cuadros dirigentes, donativos en especies y metálico, préstamos blandos, etc., etc... Todo apuntando hacia la formación, crecimiento y consolidación de la nueva derecha, como una fuerza político-social importante al interior de cada estado, con la finalidad de que, a través de su actividad, se convierta en un actor importante y llegue a formular las alternativas necesarias y suficientes, por medio de las cuales desplace a la derecha tradicional y ejerza la hegemonia a nivel económico, politico y social. Otro actor del sistema internacional, que brinda apoyo a la nueva derecha salvadorena, lo constituye su homóloga chilena. Esta última aporta la experiencia adquirida en la sociedad chilena, cuando por medio de un esquema de ajuste estructural y de liberación, lograron superar en forma parcial, la grave contracción que tuvo la economia, como consecuencia de la excesiva intervención del estado en la misma, especificamente durante el periodo del presidente Dr. Salvador Allende, situación que la nueva derecha salvadorena, juzga muy similar a la vivida en el pais, durante la época en la cual, la Democracia Cristiana, estuvo ocupando la cúpula del poder político y además se ejecutaron las reformas estructurales de todos conocidas. Reformas que la nueva derecha salvadorena, continúa considerando en la actualidad como las responsables de la crisis que se vive en todos los órdenes de la sociedad nacional. 
También se debe tener presente, que la nueva derecha salvadorena recibe asistencia directa de agencias del gobiernn estadounidense, como es el caso del AID, cuya actividad se encuentra en alguna medida orientada por los planteamientos económicos neoliberales, de la nueva derecha estadounidense.

En resumen, se puede decir que FUSADES, como la institución que representa a la nueva derecha salvadorena, recibe apoyo de agentes del sistema internacional, a nivel privado como público. Cosa que en alguna forma puede servir para inferir la importancia que revisten los planteamientos neoliberales y neoconservadores en la preservación y reproducción del sistema capitalista a nivel mundial.

Respecto al consenso que es capaz de generar las medidas particulares de los modelos a nivel económico y político-social, en las diferentes fuerzas políticas y sociales de la nación, éste se puede llegar a valorar por los efectos que se prevé producirán la puesta en práctica de tales disposiciones. En tal sentido, a nivel económico los sectores sociales favorecidos con las medidas de ajuste estructural y de liberación, manteniendo congelados los salarios, recaerá en el sector empresarial, fenómeno que consiguientemente capitalizará el apoyo del sector productivo, independientemente de que participe o no de la ideología de la nueva derecha. Los sectores sociales de ingresos medios, se verán afectados en su ingreso real, por la modificación de los precios de productos, tarifas de servicios, tipo cambio y tasas de interés..$^{\circ}$ En parte, debido a su falta de organización, o también a su tradicional patrón de conducta en apoyo de la clase fundamental, constituyen las dos causas más evidentes de la aceptación tácita de los modelos o falta de cuestionamiento a los mismos, situación que la nueva derecha hará aparecer como un nivel de consenso y apoyo a sus propuestas. Por el contrario, los sectores sociales de ingresos bajos organizados, que serán afectados directamente en el orden de sufrir una contracción real de sus salarios, por la medidas de ajuste estructural y liberalización, también constituyen la fuerza social, que, por el momento ha cuestionado con más vehemencia las medidas económicas ya ejecutadas por el gobiemo actual, y que forman parte del modelo propuesto por FUSADES.

En resumen, se puede decir que el modelo que ha propuesto FUSADES a nivel económico, podrá generar consenso en la derecha tradicional y los sectores sociales medios, no asi los sectores sociales bajos, por las razones ya expuestas. En relación al consenso capaz de generar en las diferentes fuerzas políticas y sociales, por el modelo político neoconservador propuesto por la nueva derecha salvadorefia, 
también se podrá evaluar en base de los efectos que generará. En este sentido, es de esperar que la derecha tradicional, no aceptará ni mucho menos apoyará un modelo político, que pretende desplazarla de la función de hegemonia que históricamente ha ejercido.

Consiguientemente, la derecha tradicional, aunque acepte el modelo económico, se vuelve difícil esperar que apoyará a la nueva derecha, en la ejecución del modelo político-social que propone.

Referente a la actitud que tomarán los sectores sociales de ingresos medios, en relación al modelo propuesto, se pueden prever respuestas como las siguientes: el sector que laboralmente se ubica en la posición de cuadros administrativos del sector productivo, más otros gnupos análogos, relacionados en forma directa e indirecta en algunos niveles de la operatividad de los modelos, es de esperar que apoyarán el modelo político-social de "democracia plebiscitaria", como una real altemativa de solución al conflicto político-social. El sector social medio, vinculado con el hacer académico, que sistemáticamente ha sostenido una posición de crítica al sistema, posiblemente expresará que los mecanismos de la democracia plebiscitaria, no son suficientes como para garantizar una solución satisfactoria del conflicto político-social, pues no comprende ni siquiera en forma marginal, las causas estructurales que le dieron origen. En relación al sector social de ingresos bajos organizados, que ha venido sosteniendo una posición de cuestionamiento a las medidas de los modelos que ha propuesto FUSADES, apoyándose en el argumento de que constituyen una nueva estrategia de dominación con la finalidad de mantener la injusticia y violencia estructural vigente, no los apoyará. En este panorama de respuesta, se puede llegar a decir que el modelo político-social, generará aceptación y apoyo, en el sector social de ingresos medios que se vincula con el sector productivo, no siendo asi en los restantes sectores sociales. En otros términos, el apoyo al modelo político-social, será más reducido y débil, que el consenso que generará el modelo económico.

Todo to expuesto anteriormente debe verse en una forma global y haciendo abstracción de la existencia del conflicto político-militar existente, pasemos a ver en seguida la viabilidad que pueden tener las propuestas de la nueva derecha salvadorena, en un marco de guerra civil y agudización de la misma a partir del 11 de noviembre de 1989.

\section{Vlabllidad del modelo económico neollberal}

Para evaluar la viabilidad del modelo económico propuesto por FUSADES, además de poder inferir el consenso que las políticas económicas contenidas en tal marco, pueden generar en las diferentes 
fuerzas pollticas y sociales nacionales, es preciso también conocer el objetivo que persiguen lograr. Tal objetivo la nueva derecha to sitúa en la necesidad de reactivar la economla nacional, como manera de poder corregir los graves desequilibrios macroeconómicos que aqueja. Teniendo presente esto, es prudente ver además los obstáculos que se interpondrán a la viabilidad del modelo, tanto a nivel de su ejecución como del éxito esperado. En este orden de cosas se puede sostener que, mientras la guerra interna continüe, ninguna politica económica será capaz de reactivar la economla nacional. ${ }^{10}$ Debido a razones como las siguientes: incremento de incertidumbre en la inversión, en otros términos la guerra en sl ocasiona un aumento de las expectativas negativas en el inversionista; bajos niveles de producción a nivel agricola e industrial, como consecuencia directa de la destrucción de la infraestructura económica y de la falta de condiciones adecuadas para la producción agricola, debido a que extensas zonas se encuentran en territorios conflictivos. Repercutiendo esto en la generación de un aumento del desempleo, tanto a nivel ubano como nural; la destrución de las condiciones materiales para la producción se ha proyectado provocando un aumento de los gastos de producción, fenómeno que en última instancia se ha hecho sentir hasta el consumidor, to cual, unido a la especulación y al deseo desmedido de los comerciantes en adelantar la obtención de ganancias, ha terminado en generar un incremento en la inflación. Esto, más la contracción real que ha experimentado el salario o los ingresos del sector laboral, ha incidido negativamente en el mercado intemo. También una consecuencia de la guerra, se ha expresado por medio de una disminución de los ingresos del estado originados en la tributacion fiscal, lo que, unido al incremento del gasto público en fines no productivos -mantenimiento del conflicto bélico- ha generado el crecimiento del déficit fiscal. Todo to cual ha contribuido a un deterioro mayor del sector externo, expresado en un aumento de las importaciones y una contracción de nuestras exportaciones." Fenómeno que se ha dramatizado, debido al creciente deterioro de los términos de intercambio.

Con el anterior marco de obstáculos, provocados por el conflicto militar existente en el pals, la viabilidad al modelo económico neoliberal afronta dos niveles de dificultades. El primero consiste en los problemas para la operativización de las politicas, que permitan ejecutar en la práctica las diferentes medidas especlficas del modelo. Es decir, en otros términos, la guerra es un obstáculo al funcionamiento del modelo. En otro nivel de dificultades es que la guerra también lleva casi a cero el éxito del modelo, porque, como ya se ha expresado, impide alcanzar la meta de reactivar la economia, por las razones descritas, en resu- 
men: se puede sostener que la guerra es un obstáculo, que dificulta la ejecución del modelo propuesto por FUSADES y a la vez niega las posibilidades del alcanzar las metas propuestas en el mismo.

Respecto al apoyo que se podrá esperar de las diferentes fuerzas políticas y sociales, al modelo neoliberal, éste se puede inferir en razón del beneficio a esperar, como resultado de la puesta en práctica del modelo. En este orden de cosas, las medidas económicas de ajuste estructural y liberalización ya enunciadas en la descripción del modelo. todas tienden a favorecer los intereses económicos de la derecha tradicional como la nueva derecha. Por contrario sensu, los costos de tales beneficios en favor de la clase fundamental serán pagados por los sectores sociales de ingresos medios y bajos, situación que se podrá manifestar por medio de un deterioro mayor de las condiciones de vida, de tales sectores sociales, como también por medio de un incremento de la emigración de salvadorenos. Por consiguiente, se puede inferir que el modelo económico propuesto por FUSADES, encontrará apoyo en los sectores sociales de attos ingresos, -sector empresarial a nivel industrial, agricola y de servicios- en virtud de que el modelo propuesto, el fin implicito que persigue, es la supervivencia y reproducción del sistema capitalista. Además, ninguna de las políticas que propone hace referencia a "catagorias económicas conflictivas", que impliquen cambios estructurales como podrían ser: una más equitativa distribución de la riqueza y una más justa distribución del ingreso nacional.

De tal forma que el rechazo a tal modelo, proveniente de los sectores sociales de ingresos medios y bajos, se origina, no sólo en el deterioro de las condiciones de vida que experimentarán con mayor amplitud, sino también en el hecho de que otro de los fines implicitos del modelo, estriba en la conservación del sistema capitalista, bajo el actual esquema de concentración de la riqueza y del ingreso, situación en la cual se origina la actual injusticia y violencia estructural. Por to tanto, la respuesta lógica a esperar del sector social de bajos ingresos es rechazo al modelo económico neoliberal propuesto por FUSADES.

Esto no niega que tal modelo no se lleve a la práctica y que además se haga funcionar. Más allá de eso, to que se quiere enfatizar es en el poco o ningún éxito que tendrá. Esto independientemente de que el modelo se lleve a su ejecución más que por consenso de los díferentes agentes económicos, impuesto por coacción, como ha sido el patrón de conducta de la clase fundamental en nuestro pais, a fin de poder defender y conservar sus intereses y privilegios.

De lo expuesto se puede resumir que la viabilidad del modelo económico neoliberal, se fundamentará en el poder que actualmente 
posee, tanto la nueva derecha como su hermana mayor, la derecha tradicional.

\section{Vlabilldad del modelo politico-soclal neoconservador}

La viabilidad del modelo político-social que propone la nueva derecha salvadorena, también se tiene que juzgar en función de los objetivos que persigue y además a qué fuerzas político-sociales beneficará al ejecutarse; es decir, en otros términos qué metas se propone alcanzar, y quién se propiciará de la renta política que generará. De tal manera que la meta que se propone alcanzar la nueva derecha, se puede sostener que consta de dos niveles: uno explicito formal que comprende el desarrollo de la democracia y sus instituciones, estadio político de la sociedad, a alcanzar por medio de un proceso, cuyo inicio debe ser marcado por la existencia de to que denominan como "democracia" plebiscitaria". El otro implícito real, que abarca dos hechos simultáneos: el.desplazamiento de la función de hegemonia que ha ejercido la derecha tradicional y el ejercicio de tal función por la nueva derecha, al ocupar la posición del actor desplazado.

Es lógico que, alcanzar tales metas supone vencer unos obstáculos, que se erigen como elementos que bloquean la viabilidad del modelo. Actualmente en nuestra realidad político-social, el obstáculo fundamental a la viabilidad de cualquier proyecto político-social, lo constituye el conflicto bélico interno agudizado a partir del 11 de noviembre de 1989. El conflicto bélico, aun antes de agudizarse, habia generado problemas en la escena politica nacional, que hoy se han ampliado y que se pueden resumir en los siguientes: polarización de las fuerzas politicas, situación que se manifiesta por una radicalización en las posiciones y endurecimiento de las acciones, fenómeno que se expresa por medio del discurso sectario que expresan y las acciones de violencia que ejecutan, a fin de deslegitimar al contrario y eliminarlo fisicamente. En este marco, la represión política viene a constituir una consecuencia de la polarización y un instrumento idóneo, para lograr neutralizar a la oposición; otras acciones efectuadas por el estado que apuntan en la dirección de establecer mayores condenas carcelarias en contra de presuntos miembros de la insurgencia armada - FMLN- o simples sospechosos de ser simpatizantes de tal organización político-militar, en un marco jurídico ideologizado, como son las últimas reformas propuestas al Código Procesal Penal. ${ }^{12}$ En este marco de confrontación política, que pretende, no sólo la neutralización política del adversario, sino también su eliminación física, como lo demuestra la muerte violenta de dirigentes sindicales e intelectuales, ${ }^{13}$ constituyen un conjunto de factores provocados por el conflicto militar interno, que conforman 
un poderoso obstáculo a la viabilidad del modelo pollitico-social propuesto por la nueva derecha, y que mientras no se logre la paz, mediante un proceso de diálogo con negociación, satisfactorio para todas las fuerzas políticas salvadorenas, es utópico pensar que se podrá alcanzar un desarrollo democrático en nuestro pais. Este marco de obstáculos a la viabilidad del modelo, hace dificil si no imposible alcanzar la meta de desarrollo democrático y las instituciones en que se apoyó tal estado político de la sociedad; pero esto no debe interpretarse, que la nueva derecha no llegó a ocupar la posición de hegemonla de la derecha tradicional; por el contrario, debe de verse al interior de un amplio escenario de contradicciones generado por la pugna de intereses opuestos de fracciones de clase fundamental, en lucha por ejercer la hegemonia. Ahora bien, el actor que ejerce la hegemonia, es el que se beneficia de la renta política que genera el modelo, la cual podrá cobrar expresión por medio de: influencia en el proceso de toma de decisiones políticas, privilegios especiales a nivel económico, político y social, y todas aquellas circunstancias en las que exprese de una u otra forma el nivel de poder que poseen y su disponibilidad de utilizarto, a fin de usufructuar su posición de hegemonia.

De lo expuesto se puede apreciar que los beneficios con la ejecución del modelo político-social neoconservador, será esencialmente la nueva derecha, en una escala menor, tal beneficio recaerá en la derecha tradicional, que, dentro del marco del capitalismo, en alguna forma continuará siendo un actor protagónico importante, debido a su poder económico, en virtud de la riqueza acumulada y del alto porcentaje del ingreso nacional que se apropia. En resumen: se puede decir que al pasar la nueva derecha a ocupar la posición de hegemonia, la derecha tradicional descenderá a un plano, en el cual a pesar de haber sido desplazado de la hegemonia, conservará acciones de agente protagónico y, por ende, gozará de los beneficios que le confiere tal posición. Esto no debe de servir para inferir en el sentido de que la derecha tradicional apoyará la ejecución del modelo políticosocial neoconservador; por el contrario, es de esperar oposición a la implementación de tal modelo, de parte de la derecha tradicional.

Los sectores sociales de ingresos medios y bajos, muy a pesar de los factores que constituyen y definen el esquema de la democracia plebiscitaria, es de esperar que continuarán excluidos de participar en el proceso de toma de decisiones; es decir, no ejecutarán acciones de las que se pueda inferir que han dejado de ser objeto y se han convertido en sujetos protagónicos de la política y especlficamente del proceso de toma de decisiones. Por to que se refiere al sector social laboral, es de esperar que continuará cuestionando la ejecución de tal 
modelo, en virtud de considerar que pretende mantener vigente unas relaciones políticas de dominación, sustentadas en la injusticia y violencia estructural, además de que tal estrategia neoconservadora, en ningún momento pretende alterar tal esquema en beneficio del sector laboral. Por el contrario, da la impresión de que con la praxis que propone, persigue o tiende a que se genere una ampliación y profundización de la violencia e injusticia estructural. Esto, de por sí, le resta viabilidad al modelo, porque agudizará el conflicto político-social y la guerra como su máxima expresión.

\section{Reflexiones finales}

El triunfo electoral del partido político, que representa los intereses de la derecha tradicional, en los comicios de marzo de 1989, generó expectativas de preocupación en varios sentidos: en el orden político, el discurso electoral que expresó tal actor, en el marco de una retórica dogmática y sectaria anticomunista, hacia prever que, al ocupar la cúpula del poder, se agudizaria la represión política. En el orden económico, se generaria una reversión de las reformas estructurales, al reprivatizar el comercio exterior del café, el sistema crediticio financiero y la tierra devolverla a sus antiguos propietarios. En otros términos, que los intentos por una mejor distribución de la riqueza y del ingreso, asi como de una apertura democrática, iniciados con tales reformas, en alguna medida perdian la posibilidad de continuar y consolidarse, debido a la concepción ideológica conservadora y reaccionario de dicho actor. Estas presunciones en alguna medida se empiezan a volver realidad, a partir del inicio de la gestión administrativa de dicha fuerza, cuando en forma subrepticia, se logra conocer el contenido del programa económico de gobierno de junio 1989 - diciembre 1990. ${ }^{4}$ En el que se deja claro la necesidad de revertir tales reformas, como condición sine qua non para reactivar la economia.

En este orden de cosas se puede llegar a sostener que, la retoma del control del poder político, por la derecha tradicional, en disputa con la nueva derecha, en alguna forma disminuye la viabilidad de los modelos de la nueva derecha, debido a que aquel actor privilegia las formas violentas de solución al conflicto político-social y su expresión militar en la guerra. En tal sentido, es de esperar una prolongación y ampliación del conflicto bélico interno, con un rotundo desprecio por una solución negociada del mismo. Esta es la razón por la cual, además de las ya expuestas, disminuye la viabilidad del modelo de la nueva derecha, tanto a nivel económico como político-social. En resumen y para finalizar, se puede decir que, mientras persista el conflicto bélico interno y además tienda a ampliarse e intensificarse, ningún 
proyecto económico, político y social podrá funcionar y alcanzar los objetivos que se propone.

También se debe tener claro, que todo proyecto que tienda a cambiar el actual status bólico que vive el pais, en su negociación deben participar tanto el gobiemo-F.A. como el FMLN. Pues ambos actores poseen suficiente capacidad de veto sobre los proyectos del adversario, de forma tal que no caben soluciones unilaterales ideologizadas, en las vias que puedan conducir a alcanzar la paz nacional, como requisito previo para cualquier nuevo proyecto de Nación.

\section{NOTAS}

1. Nicos Poulantzas

2. José Marln

3. FUSADES

4. Ibidem. José Marln

5. FUSADES

6. Ibidem anterior

7. Martin-Baró, Ignacio

8. Rudolf Lauff

9. Alfonso Goitia
"Poder politico y clases sociales en el estado capitalista". Editores Siglo XXI, S.A. México, D.F. 11 a edición, 1975. Págs. 117 a 146.

¿Existe un pensamiento de nueva derecha en El Salvador? Realidad económica-social. Mayo-junio/1989. No. 3. San Salvador, El Salvador, C.A. Págs. 336 a 339.

"A la conciencia de los pueblos centroamericanos y del mundo". La Prensa Gráfica, Nov. 18 de 1989. San Salvador, El Salvador, Pág, No. 19.

Op. Cit. No. 2 Págs, 336 a 339.

"La necesidad de un nuevo modelo económico para El Salvador, San Salvador, EI Salvador, 1985. Págs. 3 a 5.

Pág. 3.

"La opinión pública ante los primeros cien dias del gobierno de Cristiani". ECA No. 490-491, agosto-Sept./89. San Salvador, El Salvador, Pág. No. 721. Cuadro No. 9: "Grupo social más beneficiado con la politica económica de ARENA".

"Economia Social de Mercado". Editorial Epoca. San Salvador, El Salvador, 1986. Págs. 1 a 8.

"Los efectos de las medidas del programa económico de gobierno". Realidad económicasocial. Julio-agosto 1989. Universidad Centroamericana "José Simeón Cañas", San Salvador, El Salvador. Págs. 368 a 369. 
10. El Salvador. Proceso : Informativo semanal, Afo 9, No. 366, diciembre 1989. Contro Universitario de Documentación e Información. Universidad Controamericana "José Simeón Cahas". San Salvador, El Salvador, C.A. Págs. No. 21.

11. Ibidem. Alfonso Goitia : Pág. 367.

12. Diario "El Mundo" : San Salvador, El Salvador, C.A. Pág. No. 1 Noviembre de 1989.

13. Ibidem. "El Mundo" : Nov. de 1989. Pág. 1 y 3.

14. Programa Económico Junio 1989diciembre 1990

Fotocopia. Primer borrador para discusión técnica. San Salvador, El Salvador. Julio de1989. 